Kateryna Schöning (Leipzig)

\title{
Tradition und Innovation in der Modusanwendung in der Instrumentalmusik des 16. Jahrhunderts ${ }^{1}$
}

\section{Fragestellung und methodische Überlegungen}

Der vorliegende Beitrag behandelt ein Thema, das immer wieder zu lebhaften Diskussionen anregt. Das System der Modi, das im polyphonen Musikdenken tief eingewurzelt schien und zu einem wesentlichen Ordnungs- und Kompositionsprinzip des polyphonen Euvres des 16. Jahrhunderts entwickelt wurde, sollte damals in genuin instrumentalen Formen wie z. B. Tiento, Tokkata oder Intonation - von den Komponisten überdacht oder jedenfalls auf eine bestimmte Weise mit neuen musikalischen Ergebnissen, d. h. mit mehrstimmigen akkordischen Klangfolgen und polyphoniefreien Passagenabschnitten, in Zusammenhang gebracht werden. Infolge der stilistischen Veränderungen wäre es einerseits zu erwarten, dass die freien ${ }^{2}$ instrumentalen Formen dem Usus des Modusgebrauchs nicht ganz folgen konnten. Anzunehmen wäre auch, dass einige Merkmale des Tonartensystems weiter gepflegt wurden und die anderen eben keine Resonanz fanden. Andererseits ergibt sich die Frage, wie die Komponisten mit den Gewohnheiten der Modusanwendung umgegangen sind. Stand das Bestreben im Vordergrund, innovative Verfahren (z. B. in den norditalienischen Orgel-Tokkaten und -Intonationen das 12-tönige Glareanische Tonartensystem) aufzuarbeiten? Oder waren zurückhaltende traditionsgebundene Tendenzen (z. B. das 8tönige Tonartensystem) eher von Bedeutung? Inwiefern gingen die Komponisten den theoretischen Schemata nach? Oder umgekehrt: Orientierte sich die theoretische Grundlage an der Kompositionspraxis? Und dann: War diese Grundlage für die Instrumentalmusik spezifisch? Die Problematik ergibt sich also zunächst aus dem Phänomen der Tradition und deren praktischer Aufarbeitung in den neuen Formen der Instrumentalmusik um 16. Jahrhundert.

Die praktische Seite konkretisiert sich unter anderem in der Frage, in welcher Verbindung die aus der realen Musik erschließbaren Ergebnisse mit den von Verfassern oder Herausgebern im Titel des Stückes so akkurat angekündigten Modi standen. Das Unterscheiden zwischen dem bloßen Zuordnungsprinzip und der tatsächlich auskomponierten Grundlage wird sodann zu einem der methodisch grundsätzlichen Ansätze. ${ }^{3}$ Diese methodische Unterscheidung zwischen Theorie und Praxis ermöglicht es heute, eine binäre Betrachtungsweise $\mathrm{zu}$ formulieren und zu einem komplexen analytischen Ergebnis zu kommen, das potenziell zwei nicht widersprüchliche Befunde beinhalten kann. Zum einen kann das Stück in

1 Diese Studie entstand im Rahmen des Projektes Instrumentalgattungen vom 14. bis zum 16. Jahrhundert: Improvisation - Stil - Gattung, das durch die Alexander von Humboldt-Stiftung gefördert wurde.

2 Den Begriff „freie Formen“ verwende ich als Synonym für „improvisationsnahe Formen“. Unter dem Begriff sind entsprechend hier und im Weiteren Formen gemeint, die größtenteils aus Passagen und Akkorden bestehen und nicht durchgehend imitativ-polyphon sind. Die Bezeichnung „frei“ deutet also auf die Art der stilistischen Mittel und keineswegs auf die regellose Gestaltung der Komposition hin.

3 Das Problem wurde von Thomas Schmidt-Beste hervorgehoben: Thomas Schmidt-Beste, Art. „Modus“, in: $M G G 2$, Bd. 6, Kassel 1995, Sp. 428. 
Hinsicht auf die präskriptive Theorie betrachtet werden. Insbesondere gilt dies im kontrapunktischen Satz (auch in den Anfängen oder innerhalb der genuin instrumentalen Komposition). Dass mit dieser Methode das Tonartensystem als hypothetisch vorhandene und wirkende theoretische Basis für die genuin instrumentalen Formen im 16. und später im 17. Jahrhundert nachgewiesen werden kann, bestätigt die grundlegende Studie von Bernhard Meier. ${ }^{4}$ Hierzu gehört auch die äußere Nomenklatur der Modi. Auf der anderen Seite kann man den Blick auf die Merkmale des Stückes richten, welche der Musik inhärent sind, d. h. auf die Kriterien, die im kontrapunktfreien Satz zweifellos festzustellen sind. In den akkordisch-passagenartigen Stücken sind dies die Finalis (oder schon der Grundton eines mehrstimmigen Klanges) und der Kadenzplan des ganzen Stückes (unabhängig davon, ob die Kadenzen noch linear als Stimmkadenzen oder schon mehrstimmig harmonisch interpretiert werden). ${ }^{5}$

Die Unterscheidung der zwei Methoden begründet sich darin, dass die Kriterien der Moduslehre, wie „Stimmendisposition“, „Ambitus“ und „melodische Beschaffenheit des Initialmotivs “, 6 für akkordisch-passagenartige Kompositionen im 16. Jahrhundert nicht so aktuell waren, wie z. B. für die nach vokalem Vorbild komponierten strengen imitativen vierstimmigen Sätze in Ricercaren, Canzonen u. a. Die Analyse von Bernhard Meier bestätigt dies in vielen Tokkaten, Intonationen und Tientos. ${ }^{7} \mathrm{Zu}$ interessanten Ergebnissen kam Louis Jambou in seiner speziell dem Tiento des 16. Jahrhunderts gewidmeten Studie. Er wandte die präskriptive Methode an und stützte sich auf die Lehre von Juan Bermudo (Declaración de instrumentos musicales, Libro quarto, 1555) und Tomás de Santa Maria (Libro llamado arte de tañer fantasia, 1531-1557, gedruckt 1565). Jedoch steuerte dies seine analytischen Ausführungen zu kontrapunktisch gestalteten Tientos (bevorzugt Miguel de Fuenllana und Alonso Mudarra) und schloss freiere Tientos von Luys Milán aus. ${ }^{8}$

Die Idee, die Analyse von akkordisch-passagenartigen und imitativen Sätzen voneinander methodisch zu trennen, erwuchs freilich langsam im musikwissenschaftlichen Schrifttum. Die Abschnitte mit Passagen wurden - im Unterschied zu polyphonen - als in modaler

4 Bernhard Meier, Alte Tonarten dargestellt an der Instrumentalmusik des 16. und 17. Jahrhunderts, Kassel ${ }^{4} 2005$.

5 Dieses Analysemodell ist den Methoden von Harold S. Powers, Cristle Collins Judd und Frans Wiering gewissermaßen ähnlich. Außer dass die Methoden an der realen Musik orientiert sind, bieten sie auch im Vergleich zur Meiers Theorie jeweils eingeschränkte Moduskriterien an: z. B. für den „tonal type“ (Powers) bestimmend sind das Tonsystem, die Schlüsselung und der Grundton; für die „modal types“ (Judd) sind Exordien, Repercussa-Töne und Schlüsselung notwendig. Der entscheidende Unterschied liegt allerdings darin, dass diese Methoden explizit für die kontrapunktische vokale Musik zugeschnitten sind. Harold S. Powers, „Tonal Types and Modal Categories in Renaissance Polyphony“, in: JAMS 34/3 (1981), S. 428-470; Cristle Collins Judd, „Modal Types and Ut, Re, Mi Tonalities: Tonal Coherence in Sacred Vocal Polyphony from about 1500“, in: JAMS 45/3 (1992), S. 428-467; Frans Wiering, The Language of the Modes. Studies in the History of Polyphonic Modality, New York u. London 2001.

6 Meier, Alte Tonarten, S. 36.

7 S. z. B. die folgenden Analysen: Tientos 1, 2, 3, 5, 6, 13, 16, 21 von Antonio de Cabezón (Meier, Alte Tonarten, S. 41-42, 50, 51, 57, 59, 76-78), Tiento de IV Tono, de Falsas von Aguilera de Heredia (Ebd., S. 78); Tokkaten 1/I-5/I von Claudio Merulo (Ebd., S. 106-108), Tokkata 4 von Ercole Pasquini (Ebd., S. 113), Tokkata 2 von Sperindio Bertoldo (Ebd., S. 120-121), Tokkaten von Giovanni Maria Trabaci (Ebd., S. 140ff.); Intonationen von Andrea Gabrieli: 1, T.1-5; 2 (Ebd., S. 109); Intonationen von Giovanni Gabrieli: 5, 6 (Ebd., S. 117-118).

8 Louis Jambou, Les origines du Tiento, Paris 1982, vgl. S. 107 ff. und S. 133 ff. 
Hinsicht undeutlich bezeichnet, wie dies z. B. Arnfried Edler andeutete. ${ }^{9}$ Theodor Göllner sprach über die Freiheit der Verbindung von unkolorierten Klängen. ${ }^{10}$ Dass die genannten Kriterien in den Abschnitten und Kompositionen mit dem Übergewicht von Passagen und Akkorden mit Einschränkungen galten, vermerkte Meier:

„Dies spiegelt sich aufs deutlichste in den Ausführungen, die die Musiklehre der Renaissance den Modi einerseits und andererseits den Konsonanzen widmet: Wo von den Modi gesprochen wird, hören wir nichts von Klängen und Klangfolgen; und wo von diesen die Rede ist, vernehmen wir nichts von den Modi." 11

„Auch die allmähliche Entstehung des Dreiklangsbegriffes können wir an Zeugnissen seit etwa 1550 deutlich ablesen; und noch vor dem Jahrhundertende (1591) sieht ein deutscher Theoretiker, Cyriacus Schneegaß, im Zusammenklang von Basston, Terz und Quint erstmals ein trinitarisches Symbol. Diese ,moderne' Seite der Musik der Renaissance ist aber nicht das Ganze und geschichtlich allein ,Zählende‘."12

„Antreffen werden wir die aus dem 16. Jahrhundert uns bezeugten ,Dur-' und ,Moll-' Wirkungen im übrigen hauptsächlich dort, wo man nicht, wie im ,fugierten'Satz, vor allem Motiv-Durchführungen hört [...], sondern wo der Hörer vornehmlich Zusammenklänge wahrnimmt: also an Stellen, die im Satz nota contra notam oder in einer dieser Satzweise nahekommenden Art gehalten sind. "13

Wenden wir uns den Quellenschriften zu, so sehen wir, dass die voneinander getrennten Darstellungen vom imitativ-polyphonen und akkordisch-passagenartigen Satz im damaligen theoretischen Denken vorgeformt waren. Man erinnert sich daran, dass Girolamo Diruta seine Studien über Modi (Il Transilvano, 1609), die er von Gioseffo Zarlino, Costanzo Porta und Claudio Merulo übernehmen sollte, anhand der unbenannten imitativen Stücke und polyphonen Ricercare und Canzone und nicht der Tokkaten niederlegte. ${ }^{14}$ Tomás de Santa Maria (Libro llamado, 1565) erläuterte seine Vorstellungen der Modi auch am Beispiel der streng imitativen Stücke (der Fantasien). ${ }^{15}$ Die Abweichungen von den Regeln wurden zwar angedeutet, aber sie richteten sich nicht unmittelbar auf die freien Formen. ${ }^{16}$

9 Arnfried Edler, Gattungen der Musik für Tasteninstrumente. Teil 1: Von den Anfängen bis 1750 (= Handbuch der musikalischen Gattungen, 7,1), Laaber 1997, S. 377.

10 Theodor Göllner, Formen früher Mehrstimmigkeit in deutschen Handschriften des späten Mittelalters, Tutzing 1961, S. 87.

11 Meier, Alte Tonarten, S. 14.

12 Ebd., S. 20.

13 Bernhard Meier, „Auf der Grenze von modalem und dur-moll-tonalem System“, in: BjbHM 16 (1992), S. 58.

14 Girolamo Diruta, ,The Transylvanian' (Il Transilvano) (= Wissenschaftliche Abhandlungen, XXXVIII/2), hrsg. von Murray C. Bradshaw und Edward J. Soehnlen, Bd. II, Übers. der Ausgabe 1609, Henryville - Ottawa - Binningen 1984, S. 106, $103 \mathrm{ff}$.

15 The art of playing the fantasia (Libro Llamado El Arte de Taner Fantasia) by Fray Thomas de Sancta Maria [Valladolid, 1565], hrsg. von Almonte C. Howell und Warren E. Hultberg, Bd. I, Pennsylvania 1991, S. 192-199. Vgl. auch Macário Santiago Kastner, „La teoria de Tomás de Santa Maria comparada con la prática de algunos de sus contemporáneos“, in: Nassare 3 (1987), S.113-127.

16 Tomás vermerkt, dass es Stücke gebe, wo man den Modus nicht bestimmen oder wo einiges nicht nach den Regeln laufen könne. Tomás, El Arte de Taner, Bd I, S. 201. Diruta nannte mixti tuoni für diejenigen Stücke, die zu keinem Modus passen. Diruta, Il Transilvano, Bd. II, S. 118; Vgl. auch Faksimile Girolamo Diruta, Il Transilvano (= Bibliotheca musica Bononiensis, II, 132), Nachdr. der Ausg. 1593 und 1609, Bologna 1997, Libro II/3, Fol. 12; Paul A. Boncella, The classical Venetian organ toccata 159-1604. An ecclesiastical genre shaped by printing technologies and editorial policies, Diss. Univ. New Brunswick 1991, S. 70. 
Um nachvollziehen zu können, wie ein instrumentales Stück modal auskomponiert werden sollte, muss man von den Behauptungen ausgehen, die von der Theorie des 16. Jahrhunderts sowohl für die kontrapunktische als auch für die freie Musik deutlich postuliert wurden. Diese sind schwierig zu finden. Eine der allgemein geltenden Regeln, auf die die Theoretiker sich einigten, war allerdings diese: Jedes Stück fußt kontinuierlich vom Anfang bis zum Ende auf einer Kadenzdisposition. Auf diese Weise sollte das Stück als ein Ganzes empfunden werden. Die Diskussion ergab sich zuvörderst aufgrund der regelgerechten Kadenzordnung in den textgebundenen polyphonen Kompositionen (Pietro Aaron, Toscanello 1539). ${ }^{17}$ Die Regeln wurden weiterhin auf die zahlreichen Intabulierungen extrapoliert und schließlich jeder Art der Musik vertraut gemacht (Tomás, Libro llamado 1565 und Diruta, Il Transilvano, 1609) ${ }^{18}$ :

Transylvanian. Quando si troverà qualche Cantilena, che modularà per altre specie, che per le sue prop'ie, come se la corda finale sarà del Primo Tuono, e che la compositione sia d'altre specie composta, haverò da far giuditio sopra la corda finale, overo sopra le specie, che modula la Cantilena?

Diruta. Il dubio, che mi dimandate non è di poca importanza, atteso che si trovano delle compositioni fatte d'alcuni Compositori senza fondamento alcųno sopra le specie delli Tuoni. Questi simili si possono più tosto demandare Tuoni misti, che naturali. Quando adunque haverete da far giuditio di qual si voglia compositione, dovrete considerare bene dal principio al fine: $\&$ vedere sotto qual specie si trova composta, se sotto alle specie del primo, ò del secondo, ò di qualùnque altro Tuono, havete à guardare alle cadenze regolari, le quali danno gran lume in tal cosa; \& di poi far giuditio in qual Tuono ella sia composta; ancora achè non havesse il suo fine nella sua propria corda finale; mà fabene nella mezana, overo in qualunque altra, che tornasse bene, $\&$ più al proposito, come per esperienza si vede, che il primo Tuono terminà alle volte nella corda mezana, cioè in A, la, mi, re, come anco tutti gli altri Tuoni. E di queste compositioni se ne trovano infinite tanto nel Canto figurato quanto] nel Canto fermo: nel Canto figurato si trova quasi sempre il secondo Kyrie, overo Christe eleison terminàre nella corda mezana; \& anco la prima parte della Gloria.
Transylvanian. Wenn sich ein paar Melodien finden, die sich in anderen Spezies als ihren eigenen bewegen, als wäre der Schlussklang der erste Ton, und die Komposition in einer anderen Spezies komponiert, sollte ich dann nach dem Schlussklang („corda finale”) oder nach der Spezies, die die Melodie bestimmt, urteilen?

Diruta. Die Frage, die ihr mir stellt, ist von nicht geringer Bedeutung, schließlich gründet bei einigen Komponisten so manche Komposition nicht auf der Spezies des Tons. Diese Töne könnten eher als Toni mixti, als Toni naturali bezeichnet werden. Wenn ihr euch ein Urteil über irgendeine Komposition bilden sollt, dann prüft sie sorgfältig vom Anfang bis zum Ende: achtet darauf, unter welcher Spezies sie komponiert wurde, ob unter dem ersten, zweiten oder einem anderen Ton, berücksichtigt die regulären Kadenzen, was viel Klarheit in solchen Fällen bringt; beurteilt dann, in welchem Ton die Komposition komponiert ist; bedenkt auch, dass die Komposition nicht unbedingt auf ihrem eigenen Ton enden könnte, sondern auf einem intermediären oder irgendeinem anderen Ton, der besser passt, erfahrungsgemäß endet der erste Ton gelegentlich auf einer intermediären Note ("corda mezana"), nämlich auf $\mathrm{A}$, la, mi, re, so wie alle anderen Töne auch. Eine große Anzahl solcher Kompositionen findet sich sowohl in der Canto figurata-Musik als auch in der Canto fermo-Musik:

17 Hierzu die häufig zitierte Ausführung des Traktats, „dass man erst die Form der Tonart betrachten und die Kadenzen einrichten müsse, bevor man eine Komposition ausarbeite“. Zit. nach Italienische Musiktheorie im 16. und 17. Jahrhundert. Antikenrezeption und Satzlehre (= Geschichte der Musiktheorie, 7), hrsg. von F. Alberto Gallo, Renate Groth, Claude V. Palisca und Frieder Rempp, Darmstadt 1989, S. 118.

18 Vgl. Carl Dahlhaus, Untersuchungen über die Entstehung der harmonischen Tonalität, Kassel ${ }^{2} 1988$, S. $192 \mathrm{ff}$. 
Li Motetti, li Madrigali, quando hanno la seconda parte, la prima termina nella corda mezana. ${ }^{19}$ in der Canto figurata-Musik endet das zweite Kyrie, also das Christe eleison, fast immer mit einer Kadenz auf einer intermediären Note; ebenso wie der erste Teil des Gloria. Wenn Motetten und Madrigale einen zweiten Teil haben, endet der erste Teil auf einer intermediären Note. ${ }^{20}$

Unmittelbar zur modalen Ausformung der genuin instrumentalen Komposition äußerte sich Luys Milán (Libro de música de vihuela de mano intitulado El maestro, Valencia 1536, = Brown 15365). Das Hauptthema seiner theoretisch-praktischen Anweisungen war ebenso wie später bei Tomás die Fantasie und das Fantasieren, jedoch behandelte Milán im Unterschied zu ihm sowohl leicht polyphonisierte als auch reine akkordisch-passagenartige Fantasien. Die polyphoniefreien Fantasien betrachtete er als Synonyme zu Tientos. In seiner „Erklärung der Tonarten, die in der Vokalmusik gebräuchlich sind“ 21 beschränkte sich Milán auf folgende Kriterien: Das Stück soll richtige Clauseln und Finalis haben; es soll mit richtigen Clauseln bis zu seinem Endpunkt komponiert werden und die Hauptstimme der Komposition soll die obere Stimme sein. Dies verdeutlichte er gleich am Beispiel der freien Fantasie. ${ }^{22}$ Bemerkenswert ist, dass diese eine der ersten gedruckten Quellen der genuin instrumentalen Musik uns genaue Hinweise zu den wenigen modalen Kriterien überliefert, die zu allen (kontrapunktischen und freien) Stücken als passend betrachtet wurden und die ein Verständnis des Stückes als Ganzes gewährleisten sollten - ein Bezug, der aus den späteren Quellenschriften gänzlich verschwindet.

Inwieweit wir daher die Moduslehre in Bezug auf die akkordisch-passagenartigen Abschnitte oder Stücke analytisch zulassen können, lässt sich dem alten Schrifttum nur schwer entnehmen. Mit Sicherheit lässt sich jedenfalls feststellen, dass die akkordisch-passagenartigen Sätze eine gesonderte Position im Musikdenken des 16. Jahrhunderts eingenommen haben. Die Überlegungen zu ihrer kompositorischen Ausformung anhand der Modi gehen auf die 1530er Jahre zurück und zeigen insgesamt nur wenige Kriterien. Dies ist auch nicht verwunderlich, wenn man bedenkt, dass mit dem Aufbruch des Notendrucks seit dem Jahrhundertanfang die freien Formen die existenzielle Wahrnehmung der musikalischen Form in Gefahr brachten. Die nun schriftlich fixierte Praxis ex tempore benötigte neue Erkenntnisse, wie ein notiertes polyphoniefreies Stück komponiert werden sollte und auf welche Grundlage es sich stützte. Man benötigte eine neue Grundlage, die möglicherweise nicht gleich theoretisch begriffen werden konnte, was jedoch ihre Existenz keinesfalls in Frage stellt. Man erwartet auch nicht unbedingt, dass das Denkgerüst in Opposition zu kontrapunktischen Normen stehen würde. ${ }^{23}$

19 Diruta, Il Transilvano, Libro II/3, Fol. 3. Vgl. Tomás, El Arte de Taner, Bd I, S. 155. Die Beispiele, die Tomás zu seiner Erklärung der Kadenzen beifügt, sind imitativ, wie auch seine am Ende des zweiten Buches vorgeschlagene Fantasia a Concierto. Vgl. ebd., S. 388 f.

20 Für die Hilfe bei der Übersetzung aus dem Italienischen danke ich Elisabeth Sasso-Fruth ganz herzlich.

21 „Intelligencia y declaracion delos tonos que en la musica de canto figurato se vsan“. Luys Milán, Libro de musica de vihuela de mano intitulado El maestro (= Publikationen älterer Musik, 2), hrsg. von Leo Schrade, Wiesbaden ${ }^{2} 1967$, S. 378-380.

22 Ebd., S. 378-380.

23 Die formale Funktion der modalen Kadenzen ist eigentlich unabhängig davon zu erkennen, inwieweit das passagenartige oder/und akkordische Stück polyphon geprägt ist. Auf die formalen Funktionen der Klauseldisposition deutete Dahlhaus hin. Er meinte jedoch vokale textgebundene Cantus-firmus-Stücke. Dahlhaus, Untersuchungen, S. 192ff. Leicht angekündigt scheint das Problem in der Dissertation von Luca Bruno, wo er die Canzone villanesche betrachtet. Luca Bruno, Theory and Analysis of Harmony 
In der vorliegenden Studie werden freie Instrumentalstücke mit der angekündigten alternativen Methode betrachtet, wobei im Vordergrund die Kriterien der Finalis, der Kadenzordnung und deren Ausformung in der Komposition des ganzen Stückes stehen werden. Darüber hinaus ist es erstens notwendig, nicht nur die freien Stücke um 1600, die auf die eine oder andere Weise von den kontrapunktischen instrumentalen Traditionen geprägt wurden (in Italien z. B. ist es der Einfluss des Ricercars auf die Tokkata), sondern auch polyphoniefreie Stücke aus der ersten Hälfte des 16. Jahrhunderts zu betrachten. Für diese Studie wurden daher aus dem spanisch-italienischen Raum sowohl Tokkaten aus der Sammlung von Girolamo Diruta (Il Transilvano, I. Buch, 1593, = Brown 1593 3 ), Tokkaten von Claudio Merulo (Toccate d'intavolatura d'organo, I. und II. Buch, 1597, 1604, = Brown 1598 ; RISM M2376, M2377) und Intonationen von Andrea und Giovanni Gabrieli (Intonationi d'organo, 1593, = Brown 15934), wie auch Tientos von Luys Milán (Libro de música de vihuela de mano intitulado El maestro, Valencia 1536, = Brown 1536 ) und Alonso Mudarra (Tres libros de música en cifras para vihuela, II. Buch, Seville 1546, = Brown 1546 14 ) ausgewählt. Das Material bilden vollständig überlieferte modale Kompositionszyklen. Im Zentrum der Analyse stehen ausdrücklich imitationsfreie Kompositionen oder Stücke mit überwiegendem Anteil an polyphoniefreier Gestaltung. Um den Blick auf die Problematik zu schärfen, werden diese auch mit imitativen Kompositionen, z. B. mit Tientos von Miguel de Fuenllana (Libro de musica para vihuela, intitulado Orphenica lyra, 1554, VI. Buch, = Brown 1554 3 ) verglichen. Die Analyse richtet sich zweitens auf die ganze Komposition und nicht nur auf ihren jeweiligen Anfang. Um mich mit dem genuin instrumentalen Stück einerseits von der Seite der Theorie, und andererseits hinsichtlich der Musik selbst zu befassen, vergleiche ich die in den Überschriften vorgegebene Zyklusordnung (2.1) mit der realen Kadenzdisposition inklusive Transposition (2.2.).

\subsection{Zyklusordnung - Zuordnungsprinzip}

Die vollständig überlieferten Tiento-Zyklen von Luys Milán und Alonso Mudarra, sowie die Tokkaten von Claudio Merulo und Intonationen von Andrea und Giovanni Gabrieli zielten eindeutig auf das Erlernen der Modi. Laut der Titelangaben sollten die Kompositionen dem jeweils aktuellen Stand der theoretischen Vorschriften entsprechen und nach dem 8-tönigen oder dem 12-tönigen Moduszyklus angeordnet sein. ${ }^{24}$ Die Spanier Milán (1536) und Mudarra (1546) unterstützen hierbei das 8-tönige System, welches etwas später Juan Bermudo (Declaracion, Libro secundo, 1555) und Tomás de Santa Maria (Libro llamado, 1531-1557, gedruckt 1565) auf spanischem Gebiet ausführlich verankerten. ${ }^{25}$ Milán betrachtete die Modi paarweise, was aus seiner „Erklärung der Tonarten“ hervorgeht: Die

in Adrian Willaert's, Canzone villanesche alla napolitana (1542-1545), Diss. Università Della Calabria, 2008. Unmittelbar mit der Frage der kompositorischen Paradigmen („,compositional paradigms“) auf der Grundlage der modalen Kadenzdisposition in den imitaiv-polyphonen Tientos und Fantasien von Antonio de Cabezón und Tomás de Santa Maria beschäftigte sich Miguel A. Roig-Francolí, „Modal Paradigms in Mid-Sixteenth-Century Spanish Instrumental Composition: Theorie and Practice in Antonio de Cabezón and Tomás de Santa Maria“, in: Journal of Music Theory 38/2 (1994), S. 252 f.

24 Die Analyse der Titelbezeichnungen wurde in der Literatur vielseitig besprochen, vgl. z. B. Jambou, Les origines, S. 33 ff.; Boncella, The classical Venetian organ toccata, S. 63 ff.; Meier, Alte Tonarten, S. 3136 u. a.

25 Zum spanischen modalen System s. Jambou, Les origines, S. 31-71. 
Fantasien im 1. und 2. Modus mit der Finalis $d$ fußen auf Kadenzen $a-d$ („dlasolre“), die Fantasien im 3. und 4. Modus mit der Finalis $e$ sollten Kadenzen $a-e$ („elami“) haben, für die Fantasien im 5. und 6. Modus mit der Finalis $f$ sind Kadenzen $f-c$ (,ffaut") vorgesehen und die Fantasien im 7. und 8. Modus bleiben schließlich mit der Finalis $g$ bei Kadenzen $g-d-c($,gsolreut $) .{ }^{26}$ Milán ordnete die ganze Sammlung El Maestro der modal-zyklischen Organisation unter: Er schuf vier volle Zyklen, davon zwei aus Fantasien und jeweils einen aus Pavanen und Tientos. Die Überschriften bestätigen die theoretischen Thesen Miláns, wobei man den Eindruck gewinnt, dass er sich nicht bemühte, in den Tientos, sowie den anderen Kompositionen, authentische und plagale Modi zu unterscheiden. Er schrieb jeweils einem Tiento ein Moduspaar zu (Tabelle 3). Als eindeutiger Hinweis auf das Zuordnungsprinzip begegnen uns auch 8 Modi in den Tiento-Zyklen von Mudarra (1546) und Fuenllana (1554): Jeweils 8 Tientos sind den 8 Modi in den Titeln plakativ zugeordnet. Die authentischen und plagalen Modi sollten sich angeblich unterscheiden (Tabelle 1 und 2). An diese Tradition schließt auch eines der frühesten überlieferten Beispiele der italienischen Zyklen konsequent an - der Intonationszyklus von Andrea Gabrieli (postum 1593) (Tabelle 4).

Die späteren norditalienischen Stücke von Giovanni Gabrieli, Merulo und die aus Dirutas Sammlung sollten hingegen, gemäß der Intentionen in den Überschriften, auf dem 12-tönigen System fußen. Sie beziehen sich eher auf die Schriften von Heinrich Glarean (Dodecachordon, 1547), Gioseffo Zarlino (Le istitutioni harmoniche 1558, ${ }^{3} 1573$ ) und Diruta selbst (Il Transilvano, II. Buch, 1609). Das 12-tönige Modussystem etablierte sich allerdings mit seiner Kontroverse in Fragen der Unterscheidung des 3. und des 4. Modus sowie des 5. und des 6. Modus. ${ }^{27}$ Diruta betonte:

Il Quinto, \& il Sesto Tuono si cantano per B quadro, $\&$ questi tali li cantano, \& suonano per B molle. Io confesso d'esserni incorso in quest'errore del sesto Tuono in quella Toccata del salto cattivo, secondo la formatione delli Tuoni, e del Duodecimo Tuono trasportato alla Quinta Bassa [...]

Dico per conclusione del Quarto Tuono, che molti Compositori, \& Organisti danno il nome di Quarto Tuono à quel che è Terzo, $\&$ non sanno le modul'tioni differenti dal suo Autentico. ${ }^{28}$
Der fünfte und sechste Ton werden in B naturale gesungen, sie aber singen und spielen sie in B molle. Ich gestehe, dass mir dieser Fehler beim sechsten Ton in meiner Toccata del salto cattivo und bei der Transposition des zwölften Tones um eine Quinte tiefer unterlaufen ist [...]

Abschließend möchte ich zum vierten Ton noch anmerken, dass viele Komponisten und Organisten die Stücke nach dem vierten Ton bezeichnen, obwohl sie im dritten Ton sind, denn sie unterscheiden nicht die anderen Modulationen vom authentischen Ton.

Diruta und sein Lehrer Merulo ordneten dementsprechend die Tokkaten in 12 Tönen an, wobei in Dirutas Überschriften die Stücke in den Modi 5, 7 und 9 fehlen. Merulo bezeichnete die Tokkaten im 5. und 6. Modus doppelt als Undecimo detto Quinto Tuono und Duodecimo detto Sesto Tuono. ${ }^{29}$ Giovanni Gabrieli vereinigte den 3. und den 4. Modus in Intonationen (Tabelle 6). Diruta ergänzte dazu noch den 11. und den 12. Modus. Den Überschriften nachgehend lässt sich insgesamt feststellen, dass das 12-tönige Modussystem bis zu einem 8-tönigen (Diruta), einem 10-tönigen (Merulo) oder einem 11-tönigen (Giovanni Gabrieli) gekürzt wurde. Es ist außerdem immer wieder unterschiedlich geformt. ${ }^{30}$

26 Luys Milán, Libro de musica, S. 380.

27 Boncella, The classical Venetian organ toccata, S. $79 \mathrm{ff}$.

28 Diruta, Il Transilvano, Libro II/3, Fol. 11.

$29 \mathrm{Vgl}$. Boncella, The classical Venetian organ toccata, S. 63 und Meier, Alte Tonarten, S. 32 f.

30 Vgl. Meier, Alte Tonarten, S. 34-35, Boncella, The classical Venetian organ toccata, S. $84 \mathrm{f}$. 
Daraus kann man erschließen, dass, erstens, die Komponisten in ihren theoretischen Vorschriften (auch in der Art der Hinweise in Titeln) einem damals wohl verbreiteten Trend folgten, die Stücke in die modal angeordneten Zyklen einzuschreiben und dies zumindest als Zuordnungs-, wenn nicht als Kompositionsprinzip zu entwickeln. ${ }^{31}$ Und zweitens, dass keine einheitliche Tendenz in den Zyklusorganisationen zu sehen ist: Von Bedeutung war mehr die stützende kompositorische Funktion des Moduszyklus als die Anbindung an die konkrete Anzahl der Modi. Dies bestätigen zum Teil die Quellenschriften. Einerseits war das Modussystem eine wichtige Zuordnungsmöglichkeit in Hinsicht auf die Aufführungspraxis. Wenn die freien Formen für den Gottesdienst verfasst wurden, sollten sie an die Kirchentonarten angepasst werden: ${ }^{32}$ Giovanni Gabrieli veröffentlichte 1593 acht Intonationen seines Onkels Andrea in allen acht Tönen zusammen mit einer eigenen Fassung des Intonationenzyklus in 12 Modi. ${ }^{33}$ Eine enge Anbindung an die Praxis zeigen auch Tientos von Mudarra, welche durch den Modus an Intabulierungen von liturgischen Stücken gekoppelt waren. ${ }^{34}$ Andererseits galt es hingegen nicht als Hauptaufgabe der Tokkaten Dirutas, die Modi darzustellen, sondern die Diminutionen einzustudieren. Diruta teilte diese Aufgaben sogar in zwei zeitlich voneinander abgetrennte Bücher - Il Transilvano aus den Jahren 1593 und 1609 - auf. Von den Modi handelt das zweite Buch. Dafür schrieb Diruta auch spezielle Übungen - Modalzyklen aus Innis und Magnificats -, welche durch die zugewiesenen Psalmmelodien ihre Funktion im liturgischen Gebrauch deutlich nachweisen. Den Modalzyklus in Tokkaten ließ er also als ein selbststehendes Kompositionsprinzip, das von der liturgischen Funktion nicht unbedingt abhängig sein sollte.

\subsection{Kadenzdisposition und Transposition - Kompositionsprinzip}

Inwiefern die theoretischen Anweisungen der tatsächlichen Musik entsprachen, beobachten wir nun in den Kadenzdispositionen. In einem Modus richtig zu komponieren oder den Modus einer fertigen Komposition fehlerlos zu bestimmen, hing vor allem davon ab, ob die modalen Rahmentöne der Quint- und Quartgattungen oder Terz und Quinte über der Finalis regelhaft waren, d. h. ob "generales clausulas" und „medio clausulas" 35 auf den richtigen Tönen in der Komposition standen.

Beispielsweise standen die Kompositionen von Milán, der sich mit dem Modus theoretisch beschäftigte, der Theorie nicht so nahe wie Mudarras Tientos. Der Vergleich der Kadenzdisposition in dessen Tientos mit Bermudos und Tomás' Empfehlungen und auch mit dem Kadenzplan in Beispielen von Tomás zeigt, dass Mudarra sich streng an alle Regeln hielt (Tabelle 1). ${ }^{36}$ In Hinsicht auf die Kadenzdisposition behielt er den Unterschied zwischen den authentischen und plagalen Modi bei. ${ }^{37}$ Eine leichte Abweichung zeigen der 3. und der

31 Über die modalen Zyklen s. MGG2: „Modus“, Sp. 428-429; s. auch dort Beispiele zu Chanson-, Magnificat, Madrigal- und Ricercar-Zyklen.

32 Boncella, The classical Venetian organ toccata, S. 85.

33 Boncella meint, dass Giovanni 12 Modi mit seinen Intonationi praktisch bestätigen und damit die 8 Intonationi in 8 Modi seines Onkels Andrea Gabrieli erweitern wollte, ebd. S. 91.

34 Jambou, Les origines du Tiento, S. 97.

35 Luys Milán, Libro de musica, S. 378-380.

36 Die Tientos wurden in der Vihuela-Stimmung $A$ übertragen. S. auch die Kadenzschemata in Jambou, Les origines du Tiento, S. 115.

37 In seinem Text verzichtet Tomás, ebenso wie Milán, auf den Unterschied zwischen authentischen und plagalen Modi: es gebe gemischte Modi, Tomás, El Arte de Taner, Bd I, S. 168, 170. Doch in den No- 
4. Modus. Mudarra ging im 3. Modus noch auf die Oberquart $d$, welche, laut Tomás, zu dem 4. und nach Bermudo doch zu beiden, zum 3. und 4. Modus, gehören soll. ${ }^{38}$

Wenden wir uns nun den polyphon gestalteten Tientos von Fuenllana zu, so bemerken wir, dass in diesen Stücken noch mehrere Abweichungen auftreten (Tabelle 2). Die Aufteilung in authentische und plagale Modi blieb partiell erhalten (2., 4. und 8. Modus). Dafür korrigierte Fuenllana die Kadenzen im 1., 3. und 5. Modus. Im 1. Modus verschwindet die Kadenz auf $a$ und im 3. Modus erklingt als Finalis die Oberquart $a$ statt $e$. Das Mixolydische gewinnt außerdem im 6. und 7. Modus bevorzugte Positionen.

Diese imitativ gestalteten Tientos, von denen man erwartet, dass sie durchaus die Tonartenschemata wiedergeben, sind faktisch etwas freier komponiert als die akkordisch-passagenartigen Tientos. Die besondere Beachtung der Modus-Tradition bestätigt sich weiterhin sogar in den freien Formen, in denen die Modusnomenklatur an sich schon nicht mehr streng war. Miláns Anweisungen zu seinen Tientos könnten beispielsweise den Eindruck erwecken, dass dieser Komponist mit 8 Modi freier als Mudarra und Fuenllana umgegangen ist und die Modi bis auf 4 reduziert hat. Auch hinsichtlich seiner eigenen theoretischen Anweisungen wäre dies anzunehmen. Dennoch komponierte Milán die Kadenzdispositionen etwas rücksichtsvoller, als er es selbst beschrieb (Tabelle 3). Während die Modi 1 und 2 sowie 5 und 6 nur auf die Kadenzen der Finalis und deren Oberquint und die Modi 7 und 8 auf ein dreigliedriges Schema Finalis - Oberquart - Oberquint gründen, genau nach Miláns Vorschriften, werden die Modi 3 und 4 mit einer Kadenzdisposition Finalis - Oberquart - Obersext statt einer einfacheren Finalis - Oberquart auskomponiert. Die Kadenzen der authentischen und plagalen Kadenzschemata wurden praktischerweise vereinigt. Nicht unbeachtet blieb außerdem das seinerzeit immer seltener vorkommende Lydische.

Auf eigene Art lapidar und der Tradition verhaftet scheinen die Intonationen von Andrea Gabrieli. Die möglichen „regulären Kadenzen“39 reduzierte er bis auf Finalis-Kadenz und Oberquint-Kadenz (1., 2., 6. und 7. Modus) im 3. und 4. Modus bis auf Finalis-Kadenz und Oberquart-Kadenz (Tabelle 4). Die Intonazione del Primo Tono wurde beispielsweise durch drei Kadenzen strukturiert: auf Oberquint $a$ und zwei Mal auf Finalis $d$ (Beispiel 1, T. 5, 13 und 16). Nur der 5. und der 8. Modus blieben aufgrund der fehlenden passenden Kadenzen nicht leicht erkennbar.

tenbeispielen zu den 8 Modi und in seinen Erklärungen zu den Kadenzen ist noch eine ältere Tradition in Kraft, ebd. S. 191-199.

38 Vgl. auch Jambou, Les origines du Tiento, S. 66.

$39 \mathrm{Zu}$ den Regulären gehören Kadenzen auf allen Tönen, die innerhalb der Modi-species liegen und den Anfang, die Mitte und den Schluss der Komposition normieren: „Die als regulär bezeichneten Kadenzen sind diejenigen, die auf Tönen und intermediären Noten in der eigenen Spezies gemacht werden: die nicht-regulären Kadenzen sind solche, die außerhalb der eigenen Spezies gemacht werden und die abhängig vom kompositorischen Plan ausfallen, die regulären Kadenzen verwendet man am Anfang, in der Mitte und am Ende der Komposition (,Cantilena'), sodass die Töne ihre Eigenheit und Form nicht verlieren“ / „Le cadenze chiamate regolare sono quelle, che si fanno nelle proprie specie, delli Tuon \& anco nelle corde mezane: le irregolari sono quelle, che si fanno fuora delle proprie specie, e di queste se ne fanno seconda li propositi, che occorrononella compositione, pur che nel principio, mezo, e fine della Cantilena si faccino le sue proprie, acciò il Tuono non perda la sua propria natura, \& forma“, Diruta, Il Transilvano, Libro II/3, Fol. 3. Diese Kadenzverteilung übernahm Diruta von Gioseffo Zarlino, Das musikalische Gesamtwerk (Istitutioni harmoniche 1558-1562), Originaltext und Übers. von Christoph Hohlfeld, Bd. 4, Übersetzungsmanuskript aus der Bibliothek der Hochschule für Musik und Theater „Felix Mendelssohn Bartholdy“ Leipzig, Sign. ÜM 196, S. 89 ff. 

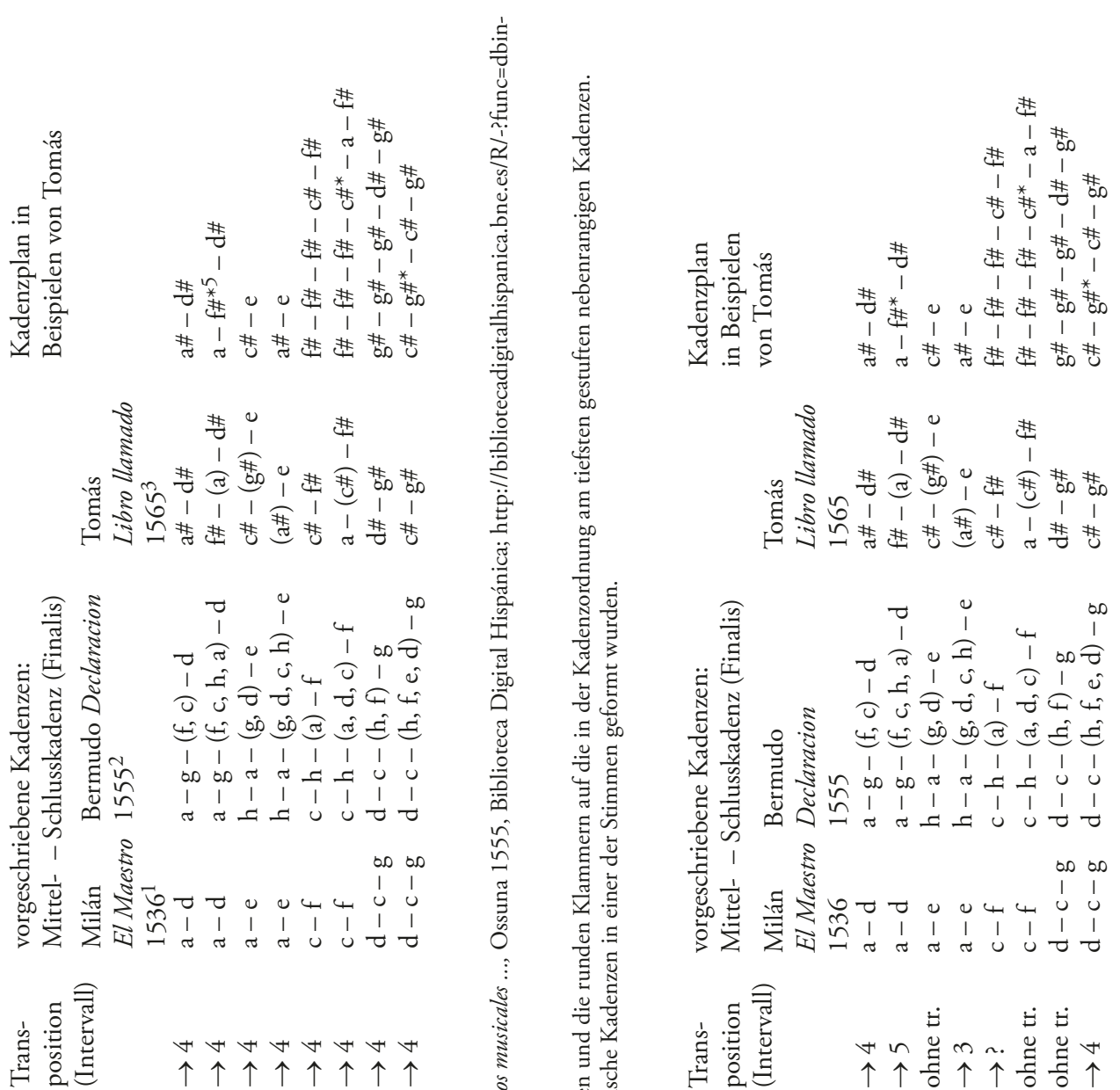

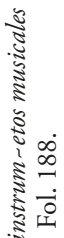


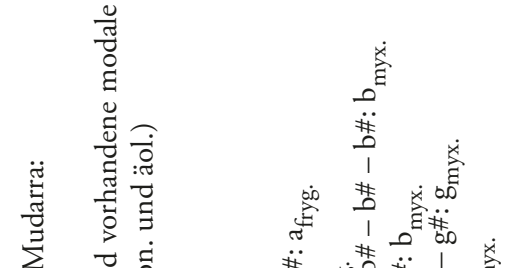

萡

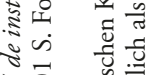
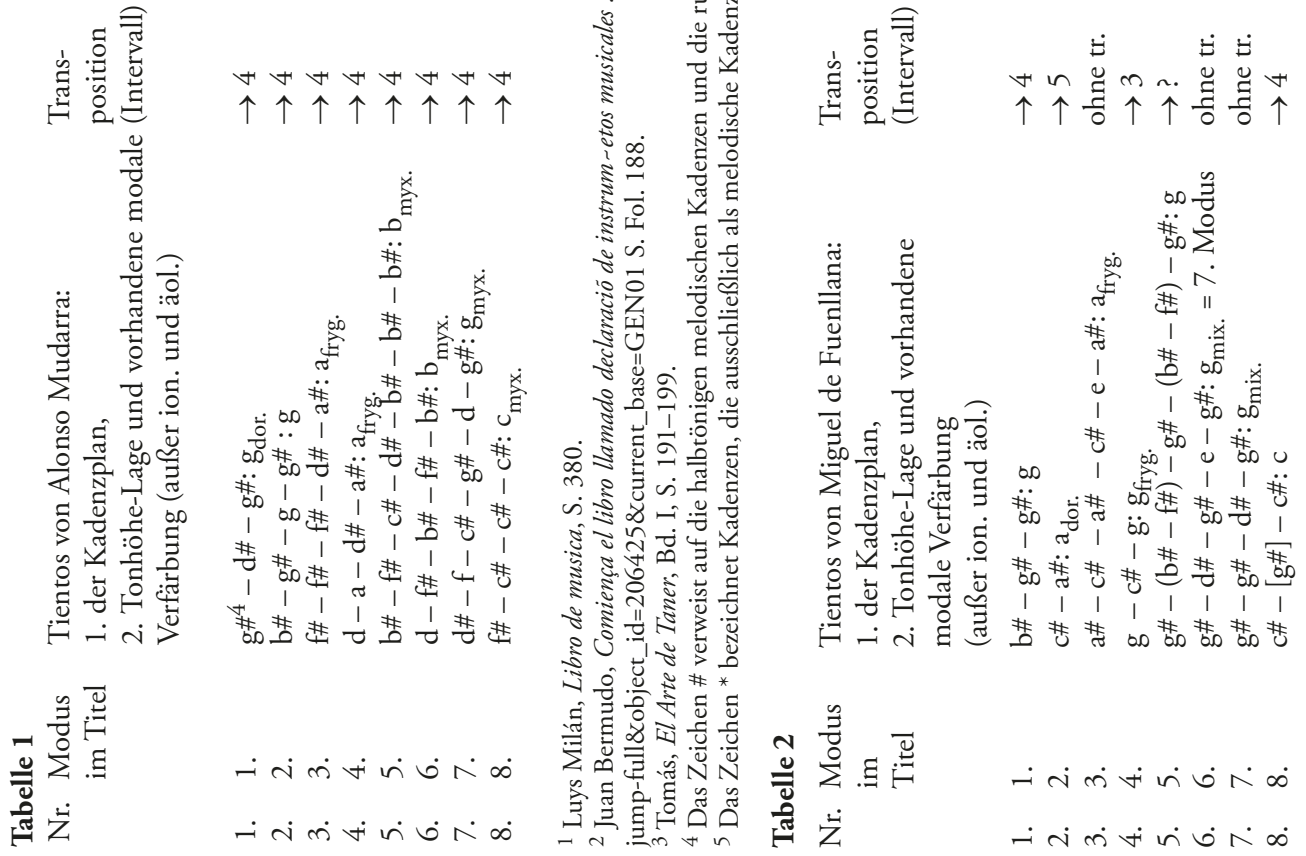

o s.믄

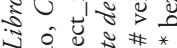

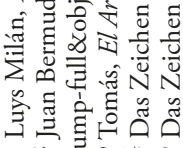

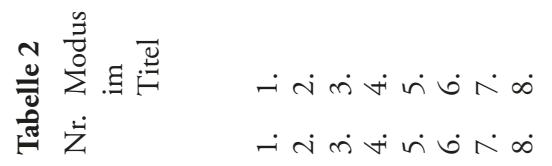



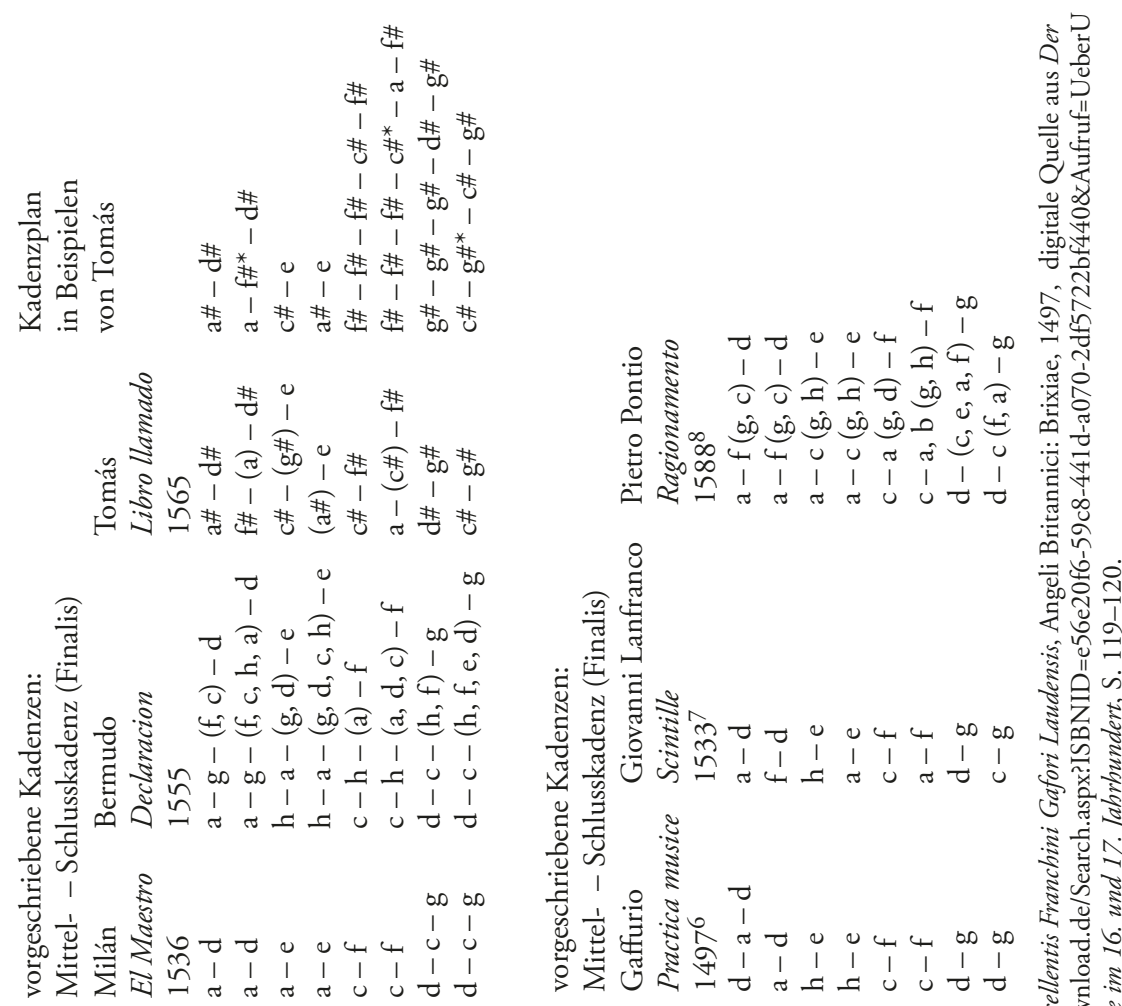

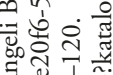

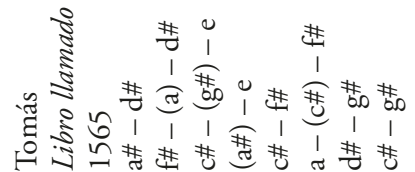

ड़े द्वे

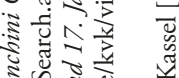



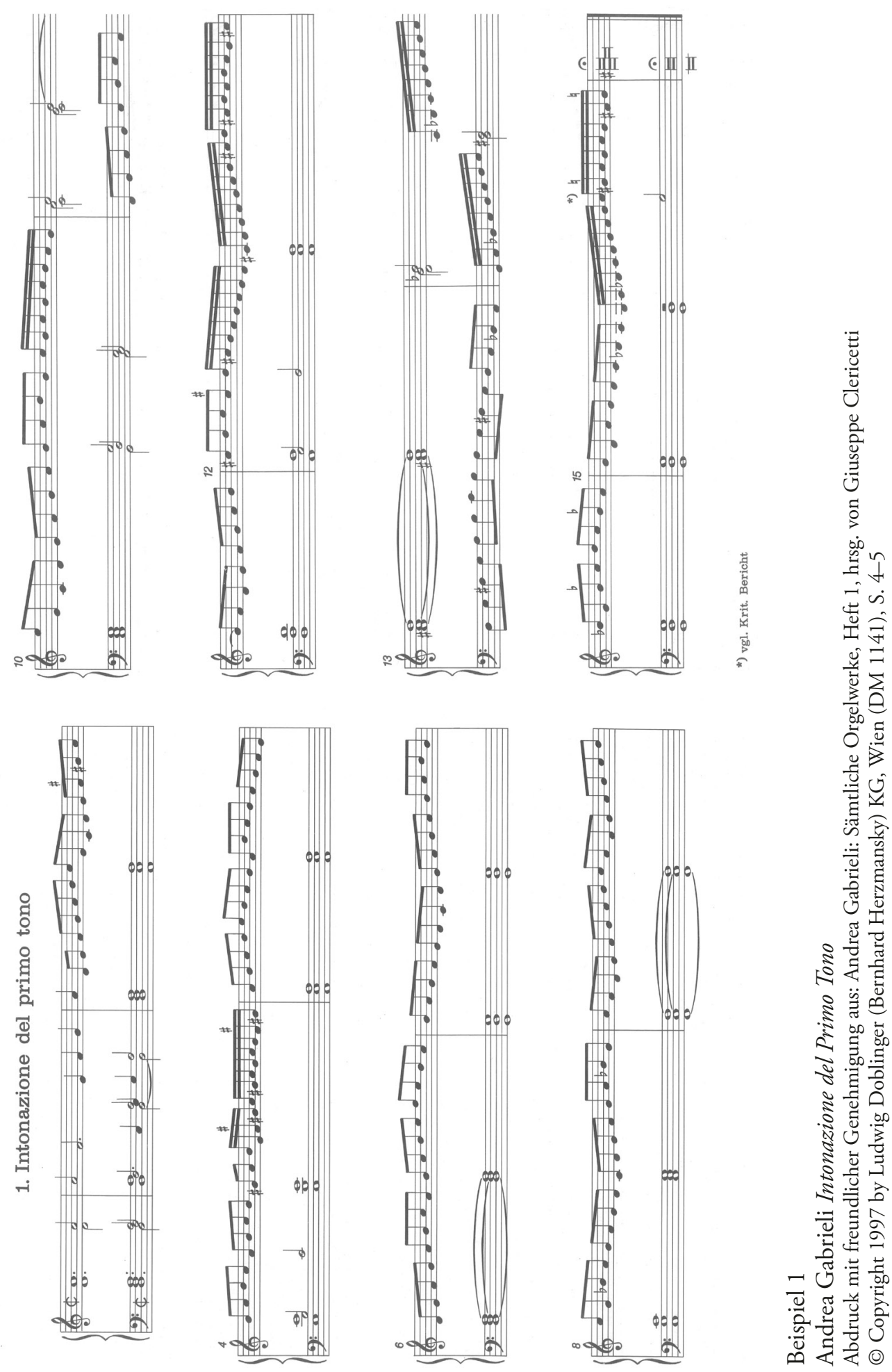
Diese auf das Nötigste reduzierte schematische Kompositionsdarstellung zeigt nicht nur eine Verringerung von Skalen, da die Stücke auf nur wenige Tonhöhe-Lagen $(d, e, c, f$ und $g$ ) fokussiert sind. Sie demonstriert eine höchst interessante Tendenz, die gerade in Italien gut nachweisbar ist: In den freien Formen wurden die Kadenzen verringert. Dabei lag das von Gabrieli demonstrierte Schema Finalis - Oberquint und für den 3. bzw. 4. Modus Finalis Oberquart zugrunde. Ausschließlich die Quinttöne als Confinalis anzugeben, geht auf die italienische Musiktheorie bis Stefano Vanneo (Recanetum, 1533) ${ }^{40}$ und weiter bis zu Franchino Gaffurio (Practica musice, 1497) ${ }^{41}$ zurück. Es handelte sich natürlich noch nicht um instrumentale Musik, und die gliedernde Funktion des Modus war - zumindest um 1500 - theoretisch noch nicht so aufgearbeitet, wie später ab Pietro Aaron (Trattato, 1525). ${ }^{42}$ Auffallend ist aber, dass die Einschränkung der Kadenztöne mit jeweils freier komponierter Musik in Verbindung stand. Eine weitere Differenzierung und komplexe hierarchische Abstufung der Kadenztöne, die die italienische Theorie reichlich nachweist, ${ }^{43}$ wurde für die freien Kompositionen nicht aufgenommen. Außer dem berühmten Beispiel der mehrstufigen Kadenz-Hierarchie bei Aaron (Trattato, 1525), entstand z. B. 1533 der Traktat Scintille von Giovanni Maria Lanfranco, mit dem gleichen Jahrgang wie Recanetum von Vanneo, in dem der Verfasser die Kadenzordnung differenzierter als Vanneo darlegte. Laut Lanfranco fundiert der Modus auf Finalis und Oberquint (1, 3, 5, 7 Modus), Finalis und Oberterz (2 und 6 Modus) und Finalis und Oberquart (4, 8 Modus) ${ }^{44}$ - eine Darstellung, die von Andrea Gabrielis Intonationen abweicht. Auch die Kadenz-Hierarchie, die kurz nach dem Tod des Komponisten öffentlich gemacht wurde - diejenige von Pietro Pontio (Ragionamento di Musica, 1588 ${ }^{45}$-, bietet, obwohl sie sehr schlicht konzipiert wurde, mehr Möglichkeiten als Gabrieli in den Intonationen genutzt hat (Tabelle 4).

Die Neigung zu einer reduzierten schematischen Darstellung der Kadenzanordnung in den freien Formen entwickelte sich beim Übergang zum 12-tönigen Tonartenschema. Hierbei sind zweierlei Formen der Behandlung der Modi zu entdecken. Von Bedeutung war nach wie vor das alte 8-tönige System. Es bildete immer noch eine Grundlage für die Intonationen und Tokkaten, auch dann noch, wenn das 12-tönige System vom Verfasser in den Überschriften der Stücke angekündigt wurde. Eine andere Tendenz bestand darin, die freien Formen an das moderne 12-tönige theoretische Konzept anzupassen und dadurch neue Wege der modalen Gestaltung zu beschreiten.

40 Stephanus Vanneus, Recanetum de musica aurea, 1533 (= Bibliotheca musica Bononiensis, II, 16), Faks., Bologna 1969; http://books.google.de/books?id=yIEyAQAAMAAJ\&printsec=frontcover\&hl=de\& source=gbs_ge_summary_r\&cad=0\#v=onepage\&q\&f=false, Buch I, Kapitel 48, Vgl. auch Italienische Musiktheorie im 16. und 17. Jahrhundert, S. $119 \mathrm{ff}$.

41 Franchino Gaffurio, Musice utriusque cantus practica: ... libris quatuor modu latissima / excellentis Franchini Gafori Laudensis, Angeli Britannici: Brixiae 1497, digitale Quelle aus Der Musikdrucken der Staats- und Stadtbibliothek Augsburg 1488-1630, http://www.fischer-download.de/Search.aspx? ISBNID=e56e20f6-59c8-441d-a070-2df5722bf440\&Aufruf=UeberUrl\&SucheTyp=Katalog\# Ergebnisse, s. Buch 1, Kapitel 8. Vgl. auch Italienische Musiktheorie im 16. und 17. Jahrhundert, S. $119 \mathrm{f}$.

42 Hierzu Italienische Musiktheorie im 16. und 17. Jahrhundert, S. 117.

43 Ebd. S. $119 \mathrm{ff}$.

44 Giovanni Lanfranco, Scintille di musica..., 1533, Digitale Quelle: http://www.ubka.uni-karlsruhe.de/ kvk/view-title/index.php?katalog=EROMM_WEBSEARCH\&url=http\%3A\%2F\%2Fgallica\%2Ebnf \%2Efr\%2Fark\%3A\%2F12148\%2Fbpt6k582203\&showCoverImg=1, S. 107.

45 Pietro Pontio, Ragionamento di musica, Faks.-Neudr. der Ausgabe 1588, hrsg. von Suzanne Clercx, Kassel [u. a.] 1959, S. 96 ff.; vgl. Italienische Musiktheorie im 16. und 17. Jahrhundert, S.119. 
Zum Beispiel: Die Kadenzdisposition in den Tokkaten aus Dirutas Il Transilvano (1593) entspricht nicht der Rangordnung der „regulären Kadenzen“, die Diruta, Zarlino folgend, im zweiten Buch seines Traktates (1609) selbst vorschlug. Als Grundlage für diese Tokkaten galten eher frühere Normen, die beispielsweise Pontio (1588) beschrieb. Einige Parallelen sind zu den Kadenz-Hierarchien von Tomás (1565) und Bermudo (1555) zu sehen (Tabelle 5 und Kadenzschemata von Bermudo und Tomás aus der Tabelle 3). Nominell stützen sich diese Tokkaten auf 12 Modi, ohne den 5., 7. und 9. Modus. Praktisch gesehen, zeigen sie hingegen die Kadenzordnungen von nur noch 8 Modi, unter denen der 1. bis 5 . Modus und 8. Modus vorhanden sind. Der 6. Modus fehlt, während der 1. und 2. sowie der 7. und 8. zusammengestellte „toni mixti“ ${ }^{46}$ bilden. Die neumodischen Modi 10, 11 und 12 lassen sich schwierig definieren, weil sie einerseits von Dirutas Musterbild abweichen und andererseits ein verbreitetes Kadenzschema Finalis - Oberquart und Finalis - Oberquint enthalten, infolge dessen diese Stücke auch in den alten Modi verstanden werden können. Toccata del XI et XII. Tuono di Girolamo Diruta (Nr. 13) hat die gleiche Kadenzordnung wie Toccata di salto cativo del Sesto Tuono di Girolamo Diruta (Nr. 3), die im 5. Modus komponiert wurde. Der 5. Modus kommt dazu in der Toccata di salto cativo del Sesto Tuono durch die Alterationen verändert vor: Es handelt sich um einen ionischen Modus auf $f$, in Analogie zu dem Ionischen auf $c$ in der Toccata Nr. 13. Die Kadenzen sind also sparsamer platziert als dies auch die älteren theoretischen Hierarchien der regulären Kadenzen von Bermudo, Tomás und Pontio zulassen.

Eine ähnliche Behandlung der Tonarten sieht man in den Intonationen von Giovanni Gabrieli (1593). Die Relikte des alten 8-tönigen Modussystems zeigen sich hier unter anderem in der Anwendung des Lydischen (Tabelle 6). ${ }^{47}$ Der 1., der 5. und der 7. Modus finden eine klare Ausprägung durch ein entsprechendes Verhältnis der Kadenzen auf Finalis und Oberquint. Dies ist aber auch eine Vereinfachung im Vergleich dazu, was Diruta in seinem Traktat verlangte. Die Kadenz-Hierarchie neigt erneut zu den früheren Kadenzordnungen, beispielsweise zu einer von Pontio (1588). Die Kadenzordnung bei Gabrieli sieht dazu auch wiederum lapidarer als in der Theorie aus. Als Basis nutzte Giovanni Gabrieli wie sein Onkel das substantielle Gerüst: Finaliston - Oberquintton. Gabrieli greift hier also auf die altüberlieferte italienische Gewohnheit, das Quintgerüst auszukomponieren, zurück. Nun geschah dies, nach Andrea Gabrieli, wiederholt in den instrumentalen freien Formen.

Parallel dazu hatte Giovanni Gabrieli Überlegungen hinsichtlich einer neuen Gestaltung eines modal angeordneten Zyklus. Das Quintgerüst übertrug er auf andere Intonationen. Das hat zur Folge, dass bei dem Versuch der Rekonstruktion die Modi durch ihre Kadenzordnung nicht mehr erkennbar sind. Der 2. Modus sieht dem 1. Modus ähnlich, erhält aber durch die Alterationen eine äolische Färbung. Die Kadenzdisposition im 6. Modus stimmt mit dem 5. Modus überein. Der 3. und 4. Modus werden in der Intonation Terzo e Quarto Tono, wie eingetragen, vereinigt, freilich mit nur einem Kadenzschema Finalis - Oberquarte. Dieses Muster mit der Quartkadenz übernahm Giovanni ebenfalls für den 9. Modus, obwohl dieser Modus, laut Dirutas Vorschriften, eine mittlere Kadenz auf der

46 Diruta, Il Transilvano, Bd. II, S. 96.

$47 \mathrm{Zu}$ den 12 Tönen in den Intonationen von Giovanni Gabrieli schrieb Bernhardt Meier: „Die von 1-12 reichende Zählung der Modi erweist sich als bloßer Schein. Was tatsächlich vorliegt, ist auch bei Giovanni Gabrieli ein System von zehn oder genauer von neun Modi, fundiert letztlich auf dem altüber-

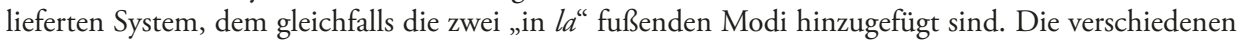
Namen, wie etwa Quinto und Undecimo Tono, Sesto und Duodecimo Tono, bezeichnen wiederum nur verschiedene Tonhöhen-Lagen", Meier, Alte Tonarten, S. 34-35. 


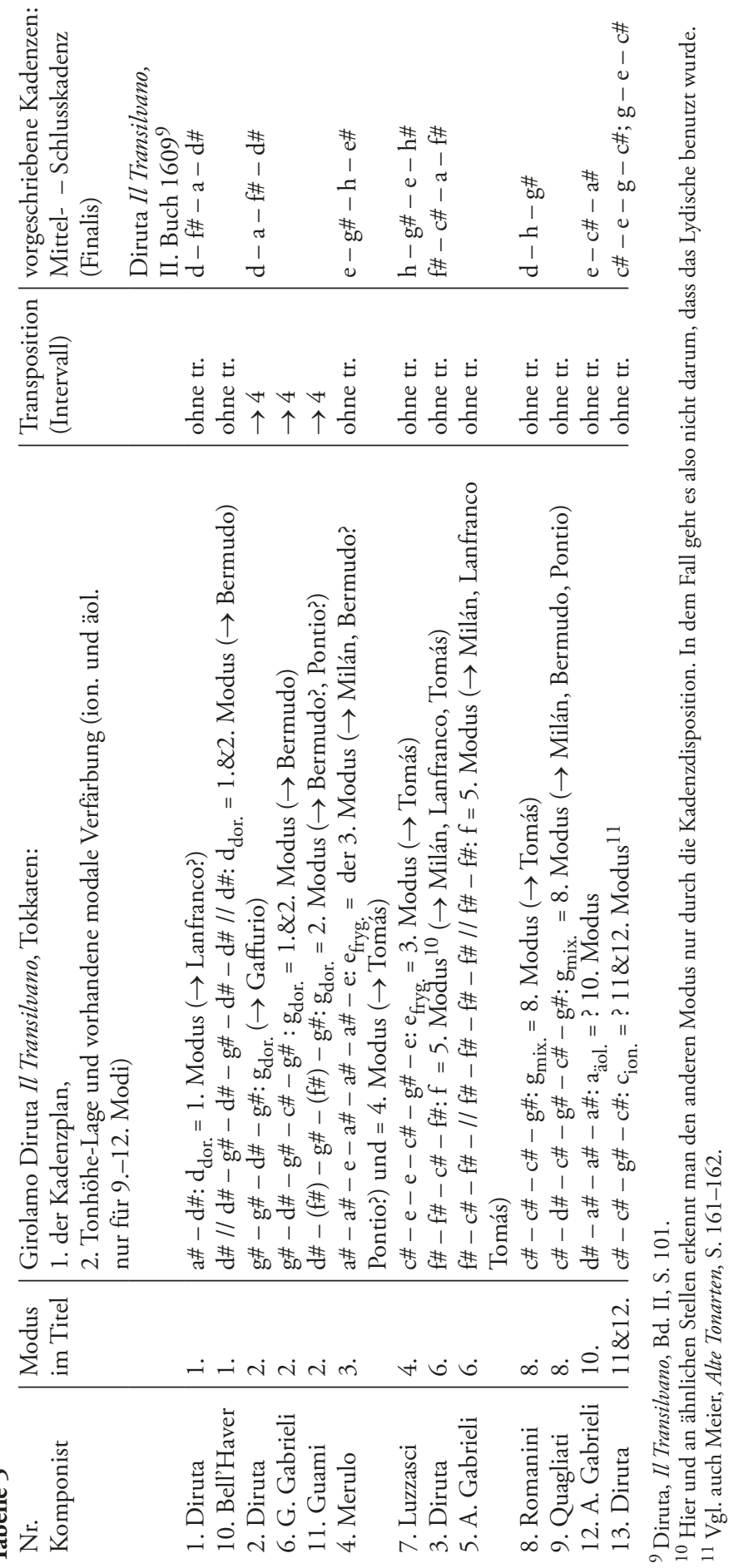


Oberquinte haben sollte. In den üblichen Modi zeichnet sich außerdem noch eine Tendenz $\mathrm{ab}$, die zukünftig immer mehr in den freien Formen zunimmt: Die Mittelkadenzen werden im fließenden Stimmengefüge versteckt, so dass der Modus sich eigentlich nur auf eine Finalis-Kadenz stützt. Dies vertuscht zunehmend die Merkmale der Tonarten und stellt ihre Existenz in Frage. Das Verfahren ist in Gabrielis Intonationen im 8., 10., 11. und 12. Modus zu beobachten. Der 8. Modus tendiert durch seine mixolydische Gestaltung zum 7. Modus; der 10. Modus wird äolisch und ist dem 9. und dem 2. Modus sehr ähnlich; der 11. und der 12. Modus sind ionisch auf $f$ und $c$, was auf Gabrielis 6. Modus zurückgeht. Insgesamt beinhalten die Intonationen von Gabrieli nur 4 nach der Kadenz-Hierarchie bestimmbare Tonarten: den 1., den 3. und 4., den 5. und den 7. Modus.

Eine kontroverse Mischung aus traditionelleren und neueren Elementen weisen die Tokkaten von Merulo $(1593,1604)$ auf. Bis hin zu Merulos Tokkaten ist anzumerken, dass die Anzahl der Kadenzen, auch der Kadenzen, die theoretische Anweisungen widerspiegeln, in den freien instrumentalen Formen um 1600 langsam steigt, obwohl die zurückhaltenden Tendenzen dominierten. Der Neigung zu den altüberlieferten Elementen folgend wäre wahrscheinlich anzunehmen, dass Merulos Tokkaten einen jüngeren Usus, also andere Traditionen als z. B. Andrea Gabrielis Intonationi, aufweisen. Seit Zarlinos Istitutioni ${ }^{3} 1573$ kam in der Musiktheorie der Brauch auf, jeden Modus in Finalis, Oberterz und Oberquint zu unterteilen. ${ }^{48}$ Diese Theorie wurzelt in Lanfrancos Ausführungen $\left(\right.$ Scintille, 1533) ${ }^{49}$ und geht später ins theoretische Denken bei Diruta ein (Il Transilvano, 1609). ${ }^{50}$ Doch folgten den Regeln weder Diruta selbst noch Merulo. Bei Merulo kann man einerseits noch deutliche Spuren der älteren Kadenz-Hierarchien sehen, z.B. der Hierarchie von Pontio (1588). Diese sieht man insbesondere gut in der Toccata Sesta dell'Terzo Tuono (I/6). ${ }^{51}$ Die FinalisKadenz auf $e$ korrespondiert mit der Kadenz des mittleren Abschnittes auf Oberquarte a\#. Der ganze Kadenzplan des Stückes erklingt nach dem folgenden Schema (fett gedruckt sind die Schlusskadenzen der größeren Abschnitte): $a \#-d \#-a \#-d \#-a \#-d \#-g \#-d \#-a \#$ $-\boldsymbol{a \#} / / \boldsymbol{a} \#-d \#-a \#-\boldsymbol{e} .{ }^{52}$ Dominant bleibt hierbei eine äolische Ausprägung. Eindeutig verkörpert sich der um eine Sekunde transponierte 7. Modus in der Toccata Sesta Settimo Tono (II/6). Es fällt andererseits auf, dass diese und andere Kadenz-Schemata von Merulo auch die Regeln der Modusbehandlung widerspiegeln, die im Laufe des 17. Jahrhunderts immer mehr reflektiert wurden (Adriano Banchieri, L’Organo suonario, 1605; Giovanni Maria Bononcini, Musico prattico, 1678). ${ }^{53}$ Eine der entscheidenden Voraussetzungen dafür war die zunehmende Reduzierung der Skalen bis zum Ionischen und Äolischen. Der 3. Modus als eine äolische Tonart in $a$ oder in $e$ war z. B. für Bononcini eine geläufige Sache. ${ }^{54}$ Der andere Modus - der 8. - zeigt sich in Merulos Toccata Settima dell' Ottavo Tono und Toccata Ottava dell'Ottavo Tuono (II/7, II/8) durch einen sich auf das Kadenzschema Finalis g\# Oberquart $c \#$ stützenden Kadenzplan, der auf den ersten Blick mit Pontios Anweisungen partiell übereinzustimmen scheint. Der ganze Kadenzplan in der Toccata Ottava dell'Ottavo

48 Zarlino, Das musikalische Gesamtwerk (Istitutioni harmoniche 1558-1562), Bd. 4, S. 54 ff.; vgl. Italienische Musiktheorie im 16. und 17. Jahrhundert, S. $118 \mathrm{ff}$.

49 Lanfranco, Scintille di musica, S. 107.

50 Diruta, Il Transilvano, Bd. II, S. 101.

51 Diese Bezeichnung bei Merulos Tokkaten verweist auf das Buch und die Nummer der Tokkata.

52 Durch das Zeichen „//“ wurden die Abschnittsgrenzen gekennzeichnet.

53 Adriano Banchieri, L'Organo suonario, Repr. der Ausgabe 1605, 1611, 1638 (= Biblioteca organologica, XXVII), Amsterdam 1969, S. 41; Italienische Musiktheorie im 16. und 17. Jahrhundert, S. 358 ff.

54 Vgl. auch Italienische Musiktheorie im 16. und 17. Jahrhundert, S. 362. 

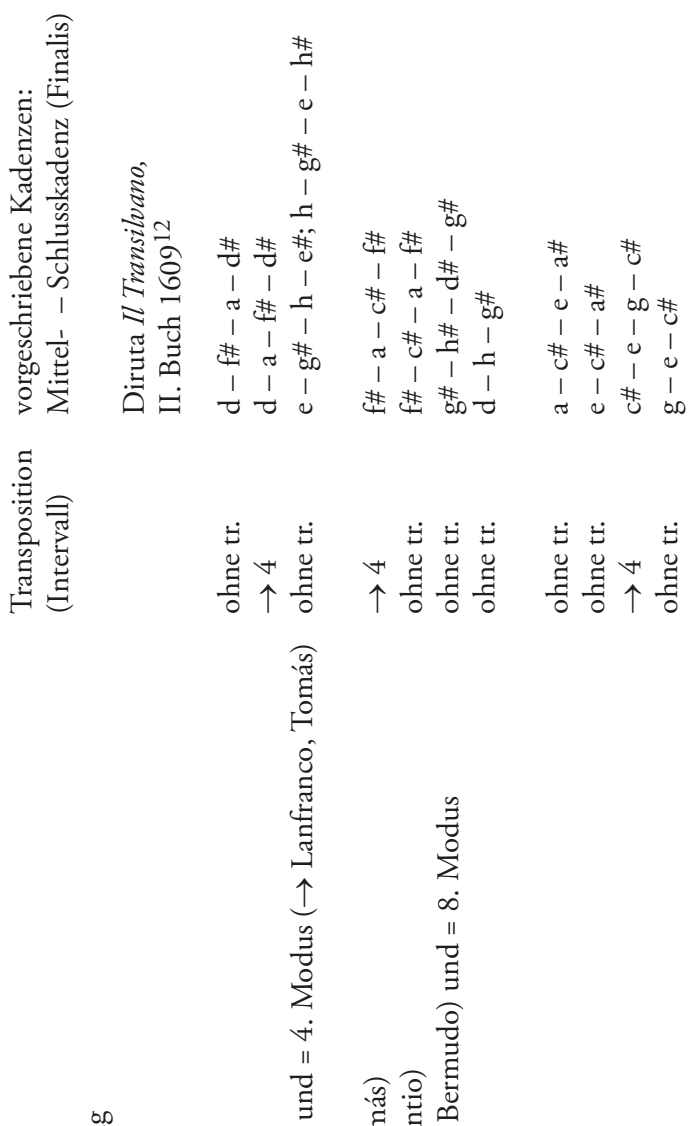

.ี

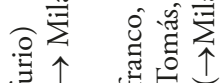

世

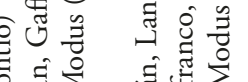

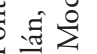

善营焉

$\uparrow \uparrow \frac{\bar{v}}{0}$

仓ิ

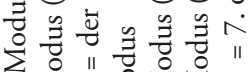

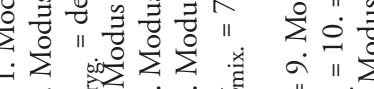

ง

चี

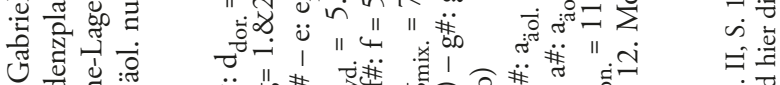

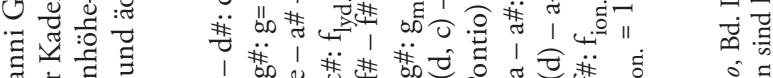

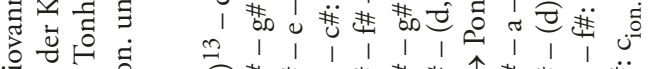

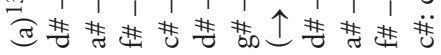

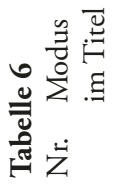




Tuono ist: $g \#-g \#-g \#-c \# / / g \#-g \#-a \#-c \#-g \# / / g \#-c \#-g \#-g \#-c \#-g \#-g \#-g \#$. Wie in der Toccata Sesta dell'Terzo Tuono gelten die den Modus bestimmenden Kadenzen als Schlusskadenzen in den größeren Abschnitten (fett gedruckt). Angesichts des herrschenden Ionischen auf $g$ neigt der Plan aber eher auch in Richtung von Bononcinis Regeln. In der Gruppe von Tokkaten im 5. und im 6. Modus präferierte Merulo den Modus in $f$ oder in $c$ per b. molle mit entsprechenden Kadenzverhältnissen $c \#$ - f\# (II/2, II/3, II/4) oder er machte den modalen Kadenzplan schwierig erkennbar (II/1). Toccata Prima Undecimo detto Quinto Tuono (II/1) ist in fper b. molle komponiert und beruht fast komplett auf der Kadenz f\#. Die Kadenzen $c$ und $b$ werden in dem Stück nur in den durchgehenden Konsonanzen umspielt: $f \#-f \#-f \#-f \#-f \#-f \#-f \#-f \#-f \#-(c \#)-f \#(b)-f \#$. Eben solche Tonarten in $f$ oder in $c$ per b. molle fungierten anstelle des 5. und 6. Modus nach Bononcini in der Praxis des 17. Jahrhunderts. ${ }^{55}$ Man findet bei Merulo schließlich immer mehr individuell gestaltete Kadenzpläne mit anderen Stufen als Basis, wie z. B. im 10. Modus in Toccata Decima del Decimo Tono (II/10) bei der Finalis-Kadenz a\# und Mittelkadenzen auf $c \#, g \#, d \#$ und $f \#$. Unter den Kadenzen an den Abschnittsgrenzen kommt allerdings immer wieder nur die Kadenz auf $a \#$ vor: $a \#-c \#-g \#-c \#-a \# / / d \#-a \#-a \# / / g \#-d \#-a \#-f \#-a-a \# / / a \#-$ $a \#-d \#-g \#-d \#-a-a \# / / a \#-a \#$. Dies erinnert wiederum an einen Hinweis Bononcinis auf den 10 . Tono als eine äolische Tonart in $a .^{56}$

Ein Zusammenhang aus den längst etablierten didaktisch-praktischen Richtlinien und weitgehenden zukünftigen Tendenzen bestätigt sich in den freien Formen durch die Transposition. Tomás und Diruta vertraten zwei verschiedene Ansichten hinsichtlich des Problems. Tomás' Meinung nach war es ungünstig oder sogar verboten, den Modus im Stück zu verlassen; ${ }^{57}$ dennoch sollte man zugleich in allen Modi spielen können. ${ }^{58}$ Er schlug eine Reihe von Tönen vor - vorwiegend die untere und obere Sekunde, obere Quarte und Quinte -, welche gelegentlich für die Transposition nutzbar sein könnten. ${ }^{59}$ Dirutas Schüler sollten aus dem Kopf zwei- bis vierstimmige Stücke nicht transponiert und dann in allen Modi transponiert spielen können:

Et per facilitarvi, farò sopra à tutti li Tuoni un Duo, $\&$ lo trasportarò in quanti luoghi si può trasportare, per poter rispondere al Choro. Prima lo dovete praticare nelli suoi tasti naturali, \& poi nelli luoghi trasportati; perche facilmente sonarete four di strada à tre, \& à quartro, tenendo l'istesso ordine, cioè sonare un Ricercare alla mente nelli tasti ordenarij, $\&$ poi sonarlo nelli luoghi trasportati. ${ }^{60}$
Damit es einfacher für euch wird, mache ich Duos in allen Tönen und transponiere sie in alle möglichen Schlüssel, so dass man dem Chor respondieren kann. Zuerst solltet ihr nicht transponiert üben (,tasti naturali“) und dann könnt ihr mit Transpositionen üben; auf diese Weise könnt ihr ganz einfach dreiund vierstimmige transponierte Stücke spielen, so wie man ein Ricercar auswendig zuerst ohne Transposition und dann transponiert spielt.

Obwohl Diruta bei der Erklärung der 12 Modi, ebenso wie Tomás, einen relativ engen Rahmen für die Transposition - auf die Oberquarte - vorgab, ${ }^{61}$ zeigte er in seinen Beispielen

55 Ebd.

56 Ebd.

57 Tomás, El Arte de Taner, Bd II, S. 96.

58 Ebd., Bd. I, S. 156; vgl. auch Otto Kinkeldey, Orgel und Klavier in der Musik des 16. Jahrhunderts, Hildesheim [u.a.] 221984, S. 54.

59 Tomás, El Arte de Taner, Bd I, S. 200.

60 Diruta, Il Transilvano, Libro II/3, Fol. 4.

61 Ebd. S. 97-99. Die Transposition um die Quarte oder die Quinte bevorzugte auch Zarlino, Das musikalische Gesamtwerk (Istitutioni harmoniche 1558-1562), Bd. 4, S. 89 ff. Zarlinos Anweisungen richten 
umfangreiche Transpositionen um Sekunde, Terz, Quarte und Quinte. ${ }^{62}$ Betrachten wir nun, wie und ob die Tientos von Mudarra, Fuenllana und Milán transponiert wurden, so sehen wir regelmäßige Transpositionen auf die Oberquarte, wie es eben Tomás bevorzugte (Tabellen 1, 2, 3). In einzelnen Fällen kommt die Oberquinte oder die bei Tomás nicht erwähnte Oberterz vor. Abgesehen davon, dass diese Transpositionen größtenteils von der Vihuela-Stimmung gefördert wurden, scheinen sie doch unabhängig davon auf das Nötigste eingeschränkt zu sein. ${ }^{63}$ Eine solche Limitierung der elementaren Aufgaben weisen auch Orgeltokkaten und -intonationen auf. Selbst Diruta vermochte die Tokkaten bis auf wenige Ausnahmen in den originalen Lagen abzudrucken (Tabelle 4). Auch Giovanni Gabrieli schrieb fast ausschließlich nicht transponierte Intonationen auf (Tabelle 5), und doch hinterließ er jeweils eine notierte Transposition um eine Quarte oder um eine Quinte auf- oder abwärts zu jeder Intonation. ${ }^{64}$ Hiermit kündigte sich eine Transpositionsordnung an, die erst um 1600 im Orgelrepertoire fest geregelt wurde. ${ }^{65}$

Zusammenfassend lässt sich feststellen, dass die freien instrumentalen Formen bis um 1600 tendenziell auf dem alten 8-tönigen Modussystem gefußt haben. Die Veränderungen des Systems bestanden in der Verringerung der Anzahl der Modi und der sehr sparsamen Anwendung der Kadenzstufen. Die modale Grundlage war in den damals neuen instrumentalen Formen meist traditioneller, als diese in den schon länger tradierten polyphonen Kompositionen. Die Berufung auf jeweils ältere modale Traditionen sorgte für die Uneinheitlichkeiten mit nominellen Modusbezeichnungen und naturgemäß mit zeitgenössischen theoretischen und praktischen Vorschriften. Angesichts der Kadenzdisposition zeigen die freien Formen ein interessantes Phänomen: Sie klammern das 12-tönige Modussystem aus ihrer Entwicklung aus, obwohl Tokkata, Intonation und Tiento ihre modale Gestaltung eigentlich der Praxis des Einsingens oder -spielens verdanken und von daher eine pedantisch klare Anwendung der Modi zeigen sollten. Erinnert man sich daran, dass die moduseigene Kadenz-Hierarchie in den Quellenschriften des 16. Jahrhunderts generell uneinheitlich und variabel war - also, wenn wir nicht nur die Traktate, die instrumentale Musik einbeziehen, berücksichtigen -, scheinen gewisse Einschränkungen auf die alten Normen in den freien instrumentalen Formen noch bemerkenswerter.

Um 1600 wechselt das altüberlieferte, im Grunde 8-tönige Tonartensystem in den freien Formen unmittelbar zu einem ebenso limitierten und praktisch nur auf wenige Tonarten fokussierten Tonartensystem des 17 . Jahrhunderts. Dies findet früher statt als das neue Tonartensystem theoretisch erschlossen wird. Die modale Kadenz-Hierarchie ist mit Ausnahme

sich aber ausschließlich auf die vokalen Formen - Motette, Madrigale, Messen, Psalmen und, laut Zarlino, auf die ,anderen Gesänge', ebd.

62 Diruta, Il Transilvano, Libro II/3, Fol. 1-6 und Libro II/4, Fol. 7-14; Kinkeldey, Orgel und Klavier, S. $130-131$.

63 Die Verbreitung der Transposition im Schrifttum des 16. Jahrhunderts hat Kinkeldey kurz zusammengefasst. Unter anderem handelt es sich um die Probleme der Orgel- und Lautenstimmung, Kinkeldey, Orgel und Klavier, S. 127-132.

64 Es handelt sich hierbei natürlich nur um das schriftlich überlieferte Repertoire. Diese Stücke könnten auch als Beispiele für die nicht notierten Übungen gelten. Auf dieser Grundlage können sich aber wissenschaftliche Spekulationen entwickeln, weil es nicht zu belegen ist. Aber wenn die Intonationen tatsächlich ein Anstoß für die Improvisationen gäben sollten, hätten die notierten Stücke doch eine privilegierte Lage, weil sie ein vorbildliches Muster bildeten.

65 Hierzu Italienische Musiktheorie im 16. und 17. Jahrhundert, S. $359 \mathrm{ff}$. 
der Finalis oft nicht mehr erkennbar und die Finalis verweist auf eine Tonhöhe-Lage und nicht auf einen Modus.

Dass die freien Formen im Laufe des 16. Jahrhunderts solche Unterschiede in dem theoretisch-praktischen Diskurs aufdecken, erklärt sich dadurch, dass der Zusammenhang von modalen Kadenzen, der den Verlauf des ganzen Stückes regelte, für die freien instrumentalen Formen bis um 1600 die einzige stützende modale Grundlage bildete. Die Finalis und die Kadenzdisposition sowie die Einschränkung auf ihre wenigen Schemata (insbesondere Finalis - Oberquint) galten als grundlegende kompositorische Basis, die immer wieder wiederholt werden musste, damit die freie instrumentale Komposition als Ganzes aufgefasst werden konnte. Ein einfaches und traditionelles Kadenzschema war eine der wesentlichen Voraussetzungen für die Überlieferung dieser Formen. Diese besonderen Funktionen der Kadenzordnung zeigen sich als ein für die Instrumentalmusik spezifisches Indiz, obwohl die Kadenzordnung sich im Allgemeinen von der vokalen Musik ableiten lässt. 